\title{
Quasar feedback revealed by giant molecular outflows
}

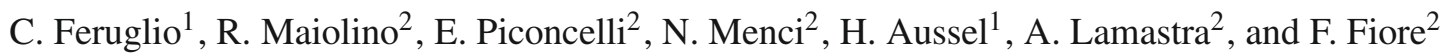 \\ ${ }^{1}$ Laboratoire AIM, DSM/Irfu/Service d'Astrophysique, CEA Saclay, 91191 Gif-sur-Yvette, France \\ e-mail: chiara.feruglio@cea.fr \\ 2 INAF - Osservatorio Astronomico di Roma, via Frascati 33, 00040 Monteporzio Catone, Italy \\ e-mail: roberto.maiolino@oa-roma.inaf.it
}

Received 6 June 2010 / Accepted 28 July 2010

\begin{abstract}
In the standard scenario for galaxy evolution young star-forming galaxies transform into red bulge-dominated spheroids, where star formation has been quenched. To explain this transformation, a strong negative feedback generated by accretion onto a central supermassive black hole is often invoked. The depletion of gas resulting from quasar-driven outflows should eventually stop star-formation across the host galaxy and lead the black hole to "suicide" by starvation. Direct observational evidence for a major quasar feedback onto the host galaxy is still missing, because outflows previously observed in quasars are generally associated with the ionized component of the gas, which only accounts for a minor fraction of the total gas content, and typically occurrs in the central regions. We used the IRAM PdB Interferometer to observe the $\mathrm{CO}(1-0)$ transition in Mrk 231, the closest quasar known. Thanks to the wide band we detected broad wings of the CO line, with velocities of up to $750 \mathrm{~km} \mathrm{~s}^{-1}$ and spatially resolved on the kpc scale. These broad CO wings trace a giant molecular outflow of about $700 M_{\odot} /$ year, far larger than the ongoing star-formation rate $\left(\sim 200 M_{\odot} /\right.$ year $)$ observed in the host galaxy. This wind will totally expel the cold gas reservoir in Mrk 231 in about $10^{7}$ yrs, therefore halting the starformation activity on the same timescale. The inferred kinetic energy in the molecular outflow is $\sim 1.2 \times 10^{44} \mathrm{erg} / \mathrm{s}$, corresponding to a few percent of the AGN bolometric luminosity, which is very close to the fraction expected by models ascribing quasar feedback to highly supersonic shocks generated by radiatively accelerated nuclear winds. Instead, the contribution by the SNe associated with the starburst fall short by several orders of magnitude to account for the kinetic energy observed in the outflow. The direct observational evidence for quasar feedback reported here provides solid support to the scenarios ascribing the observed properties of local massive galaxies to quasar-induced large-scale winds.
\end{abstract}

Key words. galaxies: active - galaxies: individual: Mrk 231 - quasars: general - galaxies: evolution

\section{Introduction}

In the standard scenario for galaxy evolution emerging from both observations and models young star-forming galaxies transform into red bulge-dominated spheroids, where star-formation has been quenched (Bell et al. 2004). Three main drivers are proposed to explain this evolution: conditions at the time of galaxy formation, galaxy interactions and mergers, and active galactic nuclei (AGN). The ubiquitous discovery of super-massive back holes (SMBHs) at the center of local bulges and the correlations between their masses and bulge properties like mass, luminosity, and velocity dispersion (Ferrarese \& Ford 2005, and references therein) suggest tight links between the evolution of AGN and their host galaxies. Indeed, massive galaxies not only live in reach environments (see e.g. Bolzonella et al. 2010; Drory et al. 2010) but are also formed in biased regions of the density field. Furthermore, galaxy mergers are more common in rich environments and are thought to destabilize cold gas, therefore enhancing star-formation and also funneling gas into the nuclear region. This gas eventually accretes onto the nuclear $\mathrm{SMBH}$, triggering an AGN. The gas and dust can intercept the line of sight to the nucleus, and therefore a natural expectation is that the early, powerful AGN phase is also highly obscured. Once SMBHs reach masses $>10^{7-8} M_{\odot}$, the AGN power is high enough to efficiently heat the gas and expel it from the galaxy through powerful winds (Silk \& Rees 1998; Fabian et al. 1999). The depletion of cold gas quenches star-formation and causes the QSO nucleus "suicide", forcing galaxies toward the $\mathrm{BH}$-spheroid mass relation observed locally. This AGN negative feedback on the star-formation in the host galaxy is thought to help in solving two long-standing problems of galaxy evolution scenarios: the observed small number of massive galaxies relative to the prediction of models (without AGN feedback) and their red colors, which are indicative of old stellar populations (Granato et al. 2004; Di Matteo et al. 2005; Menci et al. 2006, 2008; Bower et al. 2006).

While observational evidence for feedback on the intracluster medium has been observed in radio-loud AGN (Fabian et al. 2003; McNamanara \& Nulsen 2007), direct observational evidence for AGN feedback onto the gas in the host galaxy (out of which stars form) is still missing. In QSOs and nearby Seyfert nuclei prominent outflows are observed, but generally only in the ionized gas component (e.g. Crenshaw et al. 2003; Turnshek 1984; Pounds et al. 2003), which accounts only for a minor fraction of the gas mass in the host galaxy, and are generally confined in the nuclear region on pc scales or in the photoionization cones. High-velocity energetic winds, whose kinetic energy is comparable to the bolometric energy of the quasar, are observed in the galactic nuclei (Reeves et al. 2009; Moe et al. 2009; Dunn et al. 2010; Bautista et al. 2010). 
Morganti et al. (2010) reported evidence for AGN-induced massive and fast outflows of neutral $\mathrm{H}$ in powerful radio galaxies, possibly driven by the AGN jets.

The bulk of the gas in QSO hosts, i.e. the molecular phase, appears little affected by the presence of the AGN. Indeed, most studies of the molecular gas in the host galaxies of QSOs and Seyfert galaxies have found narrow $\mathrm{CO}$ lines (with a width of a few $100 \mathrm{~km} \mathrm{~s}^{-1}$ ), generally tracing regular rotation patterns, with no clear evidence for prominent molecular outflows (Downes \& Solomon 1998; Wilson et al. 2008; Scoville et al. 2003), even in the most powerful quasars at high redshift (Solomon \& Vanden Bout 2005; Omont 2007). Yet, most of the past $\mathrm{CO}$ observations were obtained with relatively narrow bandwidths, which may have prevented the detection of broad wings of the $\mathrm{CO}$ lines possibly associated with molecular outflows. Even worse, many CO surveys were performed with single dish, where broad $\mathrm{CO}$ wings may have been confused with baseline instabilities and subtracted away along with the continuum.

We present new $\mathrm{CO}(1-0)$ observations of Mrk 231 obtained with the IRAM Plateau de Bure Interferometer (PdBI). Mrk 231 is the nearest example of a quasar object and is the most luminous Ultra-Luminous Infrared Galaxy (ULIRG) in the local Universe (Sanders et al. 1988) with an infrared luminosity of $3.6 \times 10^{12} L_{\odot}$ (assuming a distance of $186 \mathrm{Mpc}$ ). A significant fraction $(\sim 70 \%)$ of its bolometric luminosity is ascribed to starburst activity (Lonsdale et al. 2003). Radio, millimeter, and nearIR observations suggest that the starbursting disk is nearly faceon (Downes \& Solomon 1998; Carilli et al. 1998; Taylor et al. 1999). In particular, past CO(1-0) and (2-1) IRAM PdBI observations of Mrk 231 show evidence for a regular rotation pattern and a relatively narrow profile (Downes \& Solomon 1998), as well as a molecular disk (Carilli et al. 1998). The existence of a quasar-like nucleus in Mrk 231 has been unambiguously demonstrated by observations carried out at different wavelengths, which have revealed the presence of a central compact radio core plus pc-scale jets (Ulvestad et al. 1999), broad optical emission lines (Lipari et al. 2009) in the nuclear spectrum, and a hard X-ray $\left(2-10 \mathrm{keV}\right.$ ) luminosity of $10^{44} \mathrm{erg} \mathrm{s}^{-1}$ (Braito et al. 2004). In addition, both optical and $X$-ray data have revealed that our line of sight to the active nucleus is heavily obscured, with a measured hydrogen column as high as $N_{\mathrm{H}}=2 \times 10^{24} \mathrm{~cm}^{-2}$ (Braito et al. 2004). The quasar Mrk 231 displays clear evidence of powerful ionized outflows by the multiple broad absorption lines (BAL) systems seen all over its UV and optical spectrum. In particular, Mrk 231 is classified as a low-ionization BAL QSOs, a very rare subclass ( $\sim 10 \%$ of the entire population) of BAL QSOs characterized by weak [OIII] emission, in which the covering factor of the absorbing outflowing material may be near unity (Boroson \& Meyers 1992). Furthermore, giant bubbles and expanding shells on kpc-scale are visible in deep HST imaging (Lipari et al. 2009). Recent observations with the Herschel Space Observatory have revealed a molecular component of the outflow, as traced by $\mathrm{H}_{2} \mathrm{O}$ and $\mathrm{OH}$ molecular absorption features (Fischer et al. 2010), but the lack of spatial information has prevented an assessment of the outflow rate.

\section{Data}

We exploited the wide bandwidth offered by the PdBI to observe the $\mathrm{CO}(1-0)$ transition in Mrk 231. The observations were carried out between June and November 2009 with the PdBI, using five of the $15 \mathrm{~m}$ antennas of the array. We observed the $\mathrm{CO}(1-0)$ rotational transition, whose rest frequency of $115.271 \mathrm{GHz}$ is redshifted to $110.607 \mathrm{GHz}(z=0.04217)$, by using using both
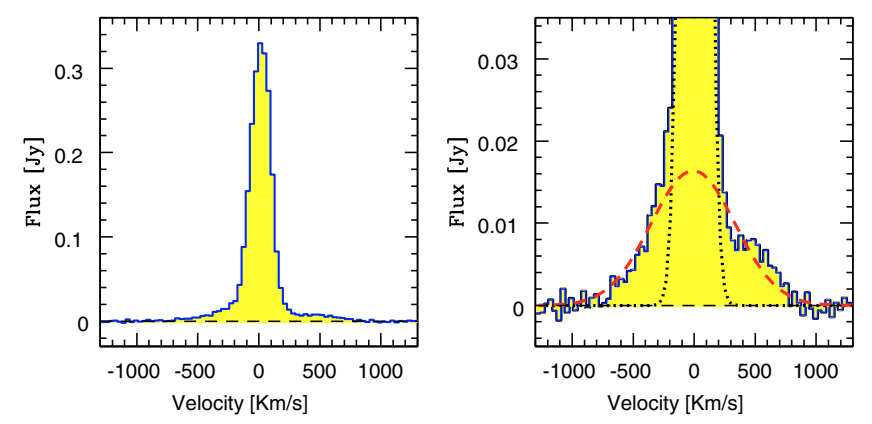

Fig. 1. Continuum-subtracted spectrum of the $\mathrm{CO}(1-0)$ transition in Mrk 231. The spectrum was extracted from a region twice the beam size (full width at half maximum, $F W H M$ ), and the level of the underlying continuum emission was estimated from the region with $v>800 \mathrm{~km} \mathrm{~s}^{-1}$ and $v<-800 \mathrm{~km} \mathrm{~s}^{-1}$. Left panel: full flux scale. Right panel: expanded flux scale to highlight the broad wings. The line profile has been fitted with a Gaussian narrow core (black dotted line) and a Gaussian broad component (long-dashed line). The FWHM of the core component is $180 \mathrm{~km} \mathrm{~s}^{-1}$ while the $F W H M$ of the broad component is $870 \mathrm{~km} \mathrm{~s}^{-1}$, and reaches a Full Width Zero Intensity (FWZI) of $1500 \mathrm{~km} \mathrm{~s}^{-1}$.

the $\mathrm{C}$ and $\mathrm{D}$ antenna configurations. The spectral correlator was configured to cover a bandwidth of about $1 \mathrm{GHz}$ in dual polarization. The on-source integration time was $\sim 20 \mathrm{~h}$. The data were reduced, calibrated channel by channel, and analyzed by using the CLIC and MAPPING packages of the GILDAS software. The absolute flux was calibrated on MWC $349(S(3 \mathrm{~mm})=$ $1.27 \mathrm{Jy})$ and $1150+497(S(3 \mathrm{~mm})=0.50 \mathrm{Jy})$. The absolute flux calibration error is of the order $\pm 10 \%$. All maps and spectra are continuum-subtracted, the continuum emission is estimated in the spectral regions with velocity $v>800 \mathrm{~km} \mathrm{~s}^{-1}$ and $v<-800 \mathrm{~km} \mathrm{~s}^{-1}$.

\section{Results}

Figure 1 shows the spectrum of the $\mathrm{CO}(1-0)$ emission line, dominated by a narrow component $\left(F W H M \sim 200 \mathrm{~km} \mathrm{~s}^{-1}\right)$, which was already detected in previous observations (Downes \& Solomon 1998; Bryant \& Scoville 1997). However, our new data reveal for the first time the presence of broad wings extending to about $\pm 750 \mathrm{~km} \mathrm{~s}^{-1}$, which have been missed, or possibly confused with the underlying continuum, in previous narrower bandwidth observations. Both the blue and red $\mathrm{CO}(1-0)$ wings appear spatially resolved, as illustrated in their maps (Fig. 2). The peak of the blue wing emission is not offset with regard to the peak of the red wing, indicating that these wings are not caused by to the rotation of an inclined disk, which leaves outflowing molecular gas as the only viable explanation. A Gaussian fit of the spatial profile of the blue and red wings (by also accounting for the beam broadening) indicates that the out-flowing medium extends over a region of about $0.6 \mathrm{kpc}\left(0.7^{\prime \prime}\right)$ in radius. To quantify the significance of the spatial extension of the high-velocity outflowing gas, we fitted the visibilities in the $u v$-plane. We averaged the visibilities of the red and blue wings in the velocity ranges $500 \div 800 \mathrm{~km} \mathrm{~s}^{-1}$ and $-500 \div-700 \mathrm{~km} \mathrm{~s}^{-1}$, and we fitted a point source, a circular Gaussian, and an inclined disk model. The results of the $u v$ plane fitting are shown in Fig. 3 and summarized in Table 1. The upper panels of Fig. 3 show the maps of the residuals after fitting a point-source model. The residuals of the red wing are $5 \sigma$ above the average rms of the map and those of the blue wing $3 \sigma$ above the rms. The lower panels of Fig. 3 show the $\mathrm{CO}(1-0)$ 

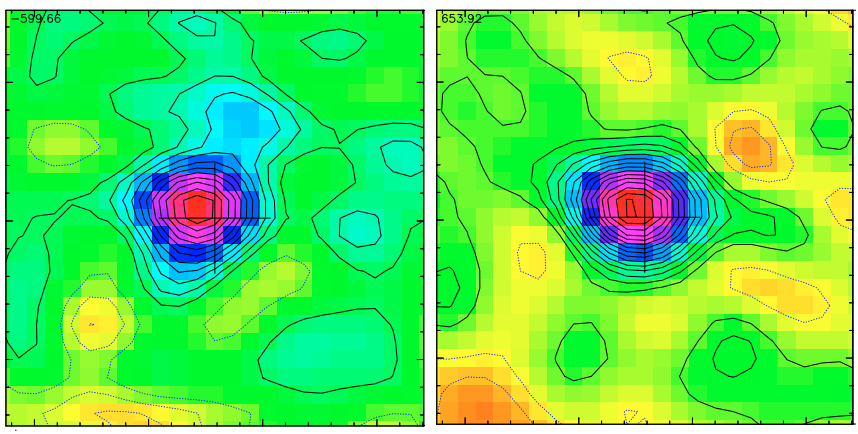

Fig. 2. $\mathrm{CO}(1-0)$ maps of the broad wings: the blue wing (left panel), integrated between -500 and $-700 \mathrm{~km} \mathrm{~s}^{-1}$ (right), and of the $\mathrm{CO}(1-0)$ red wing, integrated between 500 and $800 \mathrm{~km} \mathrm{~s}^{-1}$ (right). The size of the maps is $15^{\prime \prime}$ by $15^{\prime \prime}$. The scale is $0.64^{\prime \prime} / \mathrm{px}$. Each contour level corresponds to $0.2 \mathrm{mJy} / \mathrm{beam}$. The beam size is $2.74^{\prime \prime} \times 3.4^{\prime \prime}$. The cross indicates the peak of the radio (VLBI) emission.

wings amplitude binned in intervals of $u v$ radius, covering baselines from 10 to $200 \mathrm{~m}$. The decreasing visibility amplitudes are totally inconsistent with an unresolved source, which would instead give constant amplitudes with radius. The red symbols show the circular Gaussian model fitted to the amplitudes. The fit is not perfect, suggesting that the real spatial distribution of the red and blue $\mathrm{CO}$ wings is more complex than a simple circular Gaussian. We find that the red wing is spatially resolved with a significance of $\geq 5 \sigma$, while the blue wing is resolved only at $1.4 \sigma$. The $F W H M$ of the combined red plus blue wings is $1.42^{\prime \prime} \pm 0.2^{\prime \prime}$, corresponding to $1.2 \mathrm{kpc}$ (Table 1$)$. The inclined disk model gives major and minor axes of $3.2^{\prime \prime} \pm 0.48^{\prime \prime}$ and $1.65^{\prime \prime} \pm 0.4^{\prime \prime}$, respectively, and a position angle of $68 \pm 11 \mathrm{deg}$. We note that the extended blue-shifted emission is consistent with the result for Na I D by Rupke et al. (2005).

The determination of the mass outflow rate depends on the wind geometry and on the conversion factor, $\alpha$, between the $\mathrm{CO}$ luminosity and the molecular gas mass. We estimated the $\mathrm{CO}$ luminosity by fitting the observed line profile with a narrow plus a broad component (see Fig. 1). The integrated $\mathrm{CO}$ luminosity of the broad component is $L(\mathrm{CO})_{B}=1.16 \times 10^{9} \mathrm{~K} \mathrm{~km} \mathrm{~s}^{-1} \mathrm{pc}^{2}$, about $1 / 10$ the luminosity of the narrow component. We converted the $\mathrm{CO}$ luminosity of the broad component into molecular gas mass $M\left(\mathrm{H}_{2}\right)$ by assuming a conservative conversion factor $\alpha=0.5 M_{\odot}\left(\mathrm{K} \mathrm{km} \mathrm{s}^{-1} \mathrm{pc}^{2}\right)^{-1}$, i.e. $1 / 10$ the Galactic value. This is the lowest conversion factor found in different locations of M 82 (a typical starburst galaxy), including its molecular outflow (Weiss et al. 2001). We derive a mass of the outflowing molecular gas $M\left(\mathrm{H}_{2}\right)=5.8 \times 10^{8} M_{\odot}$, which is consistent with the lower limit of $7 \times 10^{7} M_{\odot}$ inferred by Fischer et al. (2010) based on the absorption molecular lines detected by Herschel. By assuming that this gas is uniformly distributed in a spherical volume of $0.6 \mathrm{kpc}$ in radius, its inferred density is $\sim 25 \mathrm{~cm}^{-3}$. Because the outflow velocity is at least $700 \mathrm{~km} \mathrm{~s}^{-1}$, the inferred mass outflow rate is $\mathrm{d} M\left(\mathrm{H}_{2}\right) / \mathrm{d} t=2200 M_{\odot} \mathrm{yr}^{-1}$. If we assume density profile scaling as $r^{-2}$ for the gas distribution, the inferred outflow rate is $710 M_{\odot} \mathrm{yr}^{-1}$. An alternative, even more conservative estimate of the outflow rate can be derived by ignoring the Gaussian fit of the broad CO component and by using only the luminosity directly measured from the broad wings, i.e. by integrating their flux at velocities higher than $+400 \mathrm{~km} \mathrm{~s}^{-1}$ and lower than $-400 \mathrm{~km} \mathrm{~s}^{-1}$. The luminosity of the wings is $L(\mathrm{CO})=3.2 \times$ $10^{8} \mathrm{~K} \mathrm{~km} \mathrm{~s}^{-1} \mathrm{pc}^{2}$ ( $\sim 30 \%$ of the CO luminosity of the total broad component inferred through the Gaussian fitting). In this case we obtain a lower limit on the outflow rate of $600 M_{\odot} \mathrm{yr}^{-1}$, for the
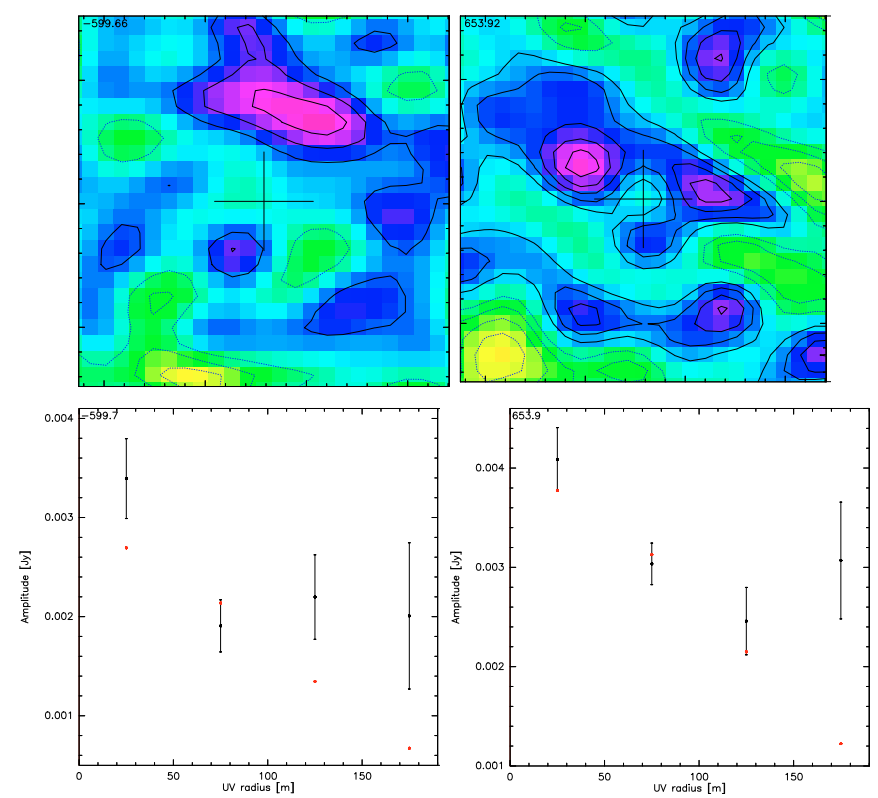

Fig. 3. Residual maps of a point-source fit to the blue (top left panel) and red (top right panel) $\mathrm{CO}$ wings. The cross indicates the position of the radio (VLBI) peak. Amplitude (in Jy) versus baseline radius for the blue (bottom left panel) and the red wings (bottom right panel). The red symbols show the results of a circular Gaussian fit (see Table 1). We recall that for an unresolved source the amplitude would be constant at all baselines.

Table 1. Integration limits, luminosity, and $u v$ fit results of CO blue and red wings.

\begin{tabular}{cccc}
\hline \hline & $\begin{array}{c}\text { Velocity range } \\
\left(\mathrm{km} \mathrm{s}^{-1}\right)\end{array}$ & $\begin{array}{c}L_{\mathrm{CO}(1-0)} \\
\mathrm{K} \mathrm{km} \mathrm{s}^{-1} \mathrm{pc}^{2}\end{array}$ & $\begin{array}{c}F W H M \mathrm{CO} \\
\left({ }^{\prime \prime}\right)\end{array}$ \\
\hline Blue wing & $-500 \div-700$ & $9.3 \mathrm{e}+07$ & $0.85( \pm 0.61)$ \\
Red wing & $500 \div 800$ & $2.3 \mathrm{e}+08$ & $1.56( \pm 0.24)$ \\
Red+Blue w. & combined & $3.2 \mathrm{e}+08$ & $1.42( \pm 0.2)$ \\
\hline
\end{tabular}

uniform gas distribution. By assuming a $\propto r^{-2}$ gas-density profile we obtain the most conservative lower limit is $\sim 260 M_{\odot} \mathrm{yr}^{-1}$. We note that alternative geometries of the outflowing wind, such as shell-like or disk-like configurations, give a higher mass outflow rate, both because the inferred outflowing gas density is higher and because the de-projected outflow velocity is higher (e.g. if the molecular outflow occurs on the galaxy disk plane). Similarly, it is easy to show that for a bipolar outflow the inferred outflow rate is the same or may be even higher (the line of sight has to intercept the outflowing molecular gas, because it is seen in absorption with Herschel, implying that the true, deprojected radius of the bipolar outflow may be equal or larger than the projected size). In any case the inferred outflow rate is much higher than the star-formation rate in the host galaxy of $200 M_{\odot} \mathrm{yr}^{-1}$ (Taylor et al. 1999; Davies et al. 2004). That the mass loss rate is much higher than the rate at which gas is converted into stars implies a phase of rapid quenching of star formation in the regions reached by the outflow $(\sim 1 \mathrm{kpc}$ scale $)$.

\section{Discussion and conclusions}

The total amount of molecular gas in the galaxy disk as inferred from the integrated emission of the narrow component, by using the $\mathrm{CO}-$ to- $\mathrm{H}_{2}$ conversion factor $\alpha$ appropriate for ULIRGs (Solomon \& Vanden Bout 2005), is $M\left(\mathrm{H}_{2}\right) \sim 10^{10} M_{\odot}$. 
Assuming the measured outflow rate of $710 M_{\odot} \mathrm{yr}^{-1}$, the total molecular gas mass will be expelled from the host galaxy within $1.4 \times 10^{7}$ years, implying the suppression of any significant star-formation activity on the same timescale. Even with the more conservative outflow rate of $260 M_{\odot} \mathrm{yr}^{-1}$ the timescale required to expel the molecular gas in the host galaxy would still be $4 \times 10^{7}$ years. This timescale is shorter than the Salpeter time $\left(\sim 5 \times 10^{7}\right.$ years $)$. We note that the quenching of starformation has probably already started in the central regions. Indeed, stellar populations younger than 5-20 $\times 10^{6}$ years are not observed within the central kpc (Lipari et al. 2009). The total kinetic power of the out-flowing gas is $1.2 \times 10^{44} \mathrm{erg} \mathrm{s}^{-1}$ (assuming an outflow rate of $700 \mathrm{~km} \mathrm{~s}^{-1}$ ), which corresponds to a few percent of the AGN bolometric luminosity, $L_{\text {Bol }} \sim 5 \times$ $10^{45} \mathrm{erg} \mathrm{s}^{-1}$ (Lonsdale et al. 2003) which, for a black hole mass of $\sim 6 \times 10^{8} M_{\odot}$ (Tacconi et al. 2002), corresponds to $6 \%$ of the Eddington luminosity. This value of the kinetic energy is very close to that expected for a shock wave produced by radiation pressure onto the interstellar medium (Lapi et al. 2005). The corresponding energy injected into the interstellar medium is $\Delta E \sim f \times L_{\mathrm{Bol}} \times t_{\mathrm{AGN}} \sim 5.5 \times 10^{57} \mathrm{erg}$, at least 4 orders of magnitude higher than the overall contribution of supernovae inferred from the observed stellar mass and age of Mrk 231 (Davies et al. 2004), and even larger than the energy injected by the radio jets (Lonsdale et al. 2003). This strongly indicates the radiation field of the QSO as the primary engine at the origin of the observed outflow. Indeed, the observed outflow velocity of $\sim 750 \mathrm{~km} \mathrm{~s}^{-1}$ implies a Mach number $M \sim 30-50$, which indicates a highly supersonic motion and, therefore, the formation of a shock front expanding in the interstellar medium of the host galaxy. Interestingly, this Mach number agrees with the expectation for the coupling between the QSO radiation field and the gas (Lapi et al. 2005). Indeed, the Mach number can be calculated as $M^{2} \sim \Delta E / E+1$, where $E=K \times T \times M_{\text {gas }} / m_{p}=3 \times 10^{54}$ erg is the thermal energy of the interstellar gas (we assumed a temperature $T=4000 \mathrm{~K}$ and an interstellar gas mass $M_{\text {gas }} \sim 10^{10} M_{\odot}$ within the shock), and $\Delta E=5.5 \times 10^{57} \mathrm{erg}$ is the overall QSO energy dumped into the interstellar medium during the time it takes the shock to reach the observed radius of $0.6 \mathrm{kpc}$. The above computation yields $M \sim 50$, which agrees well with our estimate based on the measured outflow speed. This suggests a solution to the long-standing problem of the transport of energy from the nucleus to the bulk of the galaxy: a highly supersonic shock transports outwards the energy accumulated in the center by radiatively accelerated nuclear winds. Extended emission and kinematic signatures in the $\mathrm{CO}$ line profiles similar to those observed here are predicted by hydrodynamic simulations that study the effect of AGN feedback on the molecular gas (Narayanan et al. 2006, 2008).

Owing to the simultaneous presence of a strong wind, heavy X-ray absorption, and of high star-formation rate, Mrk 231 has been regarded as one of the promising candidates of a QSO transiting from the obscured accretion phase, accompanied by vigorous star formation, where AGN feedback onto the host galaxy is in action, to the un-obscured phase (Page et al. 2004; Stevens et al. 2005) in the framework of the AGN-galaxy co-evolutionary sequence. These new observations of Mrk 231 provide one of the first direct observational evidences of QSO feedback that is dramatically affecting the evolution of its host galaxy. The QSO-driven giant molecular outflow is expected to expel the disk gas on a short time scale, therefore halting star formation. This discovery, and in particular the inferred fraction of kinetic energy injected into the ISM relative to the QSO luminosity, confirms the expectations of models predicting a tight connection between the evolution of massive galaxies and the energy released by the accreting black holes harbored in their nuclei. The greatly improved sensitivity and expanded bandwidth of current and forthcoming millimeter interferometers will allow these studies to be extended to larger samples of QSOs. Therefore, it will be possible to directly verify whether the QSO feedback onto the host galaxy is really ubiquitous or not.

Acknowledgements. We thank Arancha Castro-Carrizo and the IRAM staff in Grenoble for helping with data reduction and calibration. We are grateful to David Elbaz, Helmut Dannerbauer, and Raphael Gobat for interesting discussions. We thank the anonymous referee. We acknowledge support from grant D-SIGALE ANR-06-BLAN-01 and A-COSMOS-04-08. This work is based on observations carried out with the IRAM Plateau de Bure Interferometer. IRAM is supported by INSU/CNRS (France), MPG (Germany) and IGN (Spain).

\section{References}

Bautista, M. A., Dunn, J. P., Arav, N., et al. 2010, ApJ, 713, 25 Bell, E. F., Wolf, C., Meisenheimer, K., et al. 2004, ApJ, 608, 752 Bolzonella, M., Kovac, K., Pozzetti, L., et al. 2009 [arXiv:0907.0013] Boroson, T., \& Meyers, K. 1992, ApJ, 397, 442

Bower, R. G., Benson, A. J., Malbon, R., et al. 2006, MNRAS, 370, 645 Braito, V., Della Ceca, R., Piconcelli, E., et al. 2004, A\&A, 420, 79 Bryant, P. M., \& Scoville, N. Z. 1996, ApJ, 457, 678

Carilli, C. L., Wrobel, J. M., \& Ulvestad, J. S. 1998, AJ, 115, 928 Crenshaw, D. M., Kraemer, S. B., \& George, I. M. 2003, ARA\&A, 41, 117

Davies, R. I., Tacconi, L. J., \& Genzel, R. 2004, ApJ, 613, 781 Di Matteo, T., Springel, V., \& Hernquist, L. 2005, Nature, 433, 604 Downes, D., \& Solomon, P. M. 1998, ApJ, 507, 615

Drory, N., Bundy, K., Leauthaud, A., et al. 2009, ApJ, 707, 1595

Dunn, J. P., Bautista, M., Arav, N., et al. 2010, ApJ, 709, 611

Fabian, A. C. 1999, MNRAS, 308, 39

Fabian, A. C., Sanders, J. S., Allen, S. W., et al. 2003, MNRAS, 344, L43 Ferrarese, L., \& Ford, H. 2005, Space Sci. Rev., 116, 523

Fischer, J., Sturm, E., González-Alfonso, E., et al. 2010, A\&A, 518, L41 Granato, G. L., De Zotti, G., Silva, L., et al. 2004, ApJ, 600, 580 Lapi, A., Cavaliere, V., \& Menci, N. 2005, ApJ, 619, 60

Lipari, S., Sanchez, S. F., Bergmann, M., et al. 2009, MNRAS, 392, 1295 Lonsdale, C. J., Lonsdale, C. J., Smith, H. E., \& Diamond, P. J. 2003, ApJ, 592, 804

McNamara, B. R., \& Nulsen, P. E. J. 2007, ARA\&A, 45, 117

Menci, N., Fontana, A., Giallongo, E., et al. 2006, ApJ, 647, 753

Menci, N., Fiore, F., Puccetti, S., \& Cavaliere, A. 2008, ApJ, 686, 219

Moe, M., Arav, N., Bautista, M. A., \& Korista, K. T. 2009, ApJ, 706, 525 Narayanan, D., Cox, T. J., Robertson, B., et al. 2006, ApJ, 642, 107

Narayanan, D., Cox, T. J., Kelly, B., et al. 2008, ApJ, 176, 331

Omont, A. 2007, Rep. Prog. Phys., 70, 1099

Page, M. J., Stevens, J. A., Ivison, R. J., \& Carrera, F. J. 2004, ApJ, 611, L85

Pounds, K. A., Reeves, J. N., King, A. R., et al. 2003, MNRAS, 345, 705

Reeves, J., O’Brien, P. T., Braito, V., et al. 2009, ApJ, 701, 493

Rupke, D. S., Veilleux, S., \& Sanders, D. B. 2005, ApJ, 632, 751

Sanders, D. B., Soifer, B. T., Elias, J. H., et al. 1988, ApJ, 325, 74

Scoville, N. Z., Frayer, D. T., Schinnerer, E., \& Christopher, M. 2003, ApJ, 585, L105

Silk, J., \& Rees, M. J. 1998, A\&A, 331, L1

Solomon, P. M., \& Vanden Bout, P. A. 2005, ARA\&A, 43, 677

Stevens, J. A., et al. 2006, MNRAS, 360, 610

Tacconi, L. J., Genzel, R., Lutz, D., et al. 2002, ApJ, 580, 73

Taylor, G. B., Silver, C. S., Ulvestad, J. S., \& Carilli, C. L. 1999, ApJ, 519, 185

Turnshek, D. A. 1984, ApJ, 280, 51

Ulvestad, J. S., Wrobel, J. M., \& Carilli, C. L. 1999, ApJ, 516, 127

Weiss, A., Neininger, N., Huttemeister, S., \& Klein, U. 2001, A\&A, 365, 571

Wilson, C. D., Petitpas, G. R., Iono, D., et al. 2008, ApJ, 178, 189 